\title{
Understanding the Effects of Structured Note-taking Systems for Video-based Learners in Individual and Social Learning Contexts
}

\author{
JINGCHAO FANG, University of California, Davis, USA \\ YANHAO WANG, Zhejiang University, China \\ CHI-LAN YANG, The University of Tokyo, Japan \\ CHING LIU, National Tsing Hua University, Taiwan \\ HAO-CHUAN WANG, University of California, Davis, USA
}

Video-based learning is widely adopted by online learners, yet, learning experience and quality may be negatively affected by asynchronous and remote natures of video-based learning. As note-taking is a common practice employed by video-based learners and is known to be an effective way to trigger active construction and processing of knowledge, yet as a meta-skill, it is challenging to most learners. In this study, we aim to approach the goal of providing cognitive and social scaffolds to video-based learners by structuring their note-taking process. We presented and evaluated structured note-taking systems designed for learners in two contexts, namely, individual learning context and social learning context. With an online controlled study involving 43 participants, we compared the structured note-taking systems with two baseline systems (for individual learning and social learning contexts respectively) and found that structured note-taking significantly improved certain aspects of video-based learning such as and higher cognitive engagement and lower distraction. We discussed our results to inform the design, iteration, and adoption of note-taking tools in video-based learning.

CCS Concepts: $\bullet$ Human-centered computing $\rightarrow$ Collaborative and social computing systems and tools.

Additional Key Words and Phrases: video-based learning; online learners; digital note-taking; cognitive engagement

ACM Reference Format:

Jingchao Fang, Yanhao Wang, Chi-Lan Yang, Ching Liu, and Hao-Chuan Wang. 2022. Understanding the Effects of Structured Note-taking Systems for Video-based Learners in Individual and Social Learning Contexts. Proc. ACM Hum.-Comput. Interact. 6, GROUP, Article 21 (January 2022), 21 pages. https://doi.org/10.1145/3492840

\section{INTRODUCTION}

Video-based learning has become widely employed in the past decade. Besides learners who primarily rely on massive open online courses (MOOC) which grow rapidly and attract learners from all over the world, students in traditional schools and universities may also experience a mix of online distance learning and face-to-face learning [23] as institutes increasingly provide students

Authors' addresses: Jingchao Fang, jcfang@ucdavis.edu, University of California, Davis, Davis, California, USA; Yanhao Wang, wangyanhao@zju.edu.cn, Zhejiang University, Zhejiang, China; Chi-Lan Yang, chilan.yang@cyber.t.u-tokyo.ac.jp, The University of Tokyo, Tokyo, Japan; Ching Liu, chingliu@gapp.nthu.edu.tw, National Tsing Hua University, Hsinchu, Taiwan; Hao-Chuan Wang, hciwang@ucdavis.edu, University of California, Davis, Davis, California, USA.

Permission to make digital or hard copies of all or part of this work for personal or classroom use is granted without fee provided that copies are not made or distributed for profit or commercial advantage and that copies bear this notice and the full citation on the first page. Copyrights for components of this work owned by others than ACM must be honored Abstracting with credit is permitted. To copy otherwise, or republish, to post on servers or to redistribute to lists, requires prior specific permission and/or a fee. Request permissions from permissions@acm.org.

(c) 2022 Association for Computing Machinery.

2573-0142/2022/1-ART21 \$15.00

https://doi.org/10.1145/3492840

Proc. ACM Hum.-Comput. Interact., Vol. 6, No. GROUP, Article 21. Publication date: January 2022. 
opportunities to enroll in blended learning programs. Unquestionably, asynchronous video-based learning has many advantages over the traditional model of teaching and learning. For example, students are able to learn by watching pre-recorded lectures with greater flexibility such that they may take classes at any time and anywhere.

Nevertheless, such asynchronous and remote nature of video-based learning also introduces challenges. For example, video-based learners may suffer from distraction and have difficulty sustaining attention and engaging in the course content, which can negatively affect academic performance $[1,7,45,46]$. In traditional classes, instructors can utilize strategies such as interactive questioning and answering to break students' mind-wandering and guide their attention to the important topics, as well as acquiring students' level of engagement in real-time and make adjustments accordingly [17]. However, the absence of interaction and communication between instructors and students makes video-based learning a form of self-regulated learning in which students themselves are responsible for managing and sustaining the learning process and regulate their learning quality, and little external scaffold exists for heightening attention and improving students' engagement $[4,46,55]$.

Note-taking is a common practice that learners perform to actively engage themselves with learning activities [16], yet, note-taking during learning requires multitasking that's cognitively demanding and may become one source of distraction. Studies showed that note-taking, though widely believed to be beneficial, may also have detrimental effects on learning [21], especially when learners employ impaired note-taking strategies. Challenges to note-taking identified include taking incomplete notes that are limited for future use [21]; taking verbatim notes (e.g., copy-and-paste from the lectures or textbooks) which does not trigger in-depth knowledge processing [3, 33]; and taking full notes and put in significant attention and reasoning effort, but being distracted from the lecture content and failed to process rapid and dense lecture presentations [19].

Recent work has started to explore how to provide interface guidance and structured workflows to support learners taking longer and no-verbatim notes, especially in the context of online education [32]. In online video-based learning, we identified the opportunity of structuring the note-taking process for video-based learners as an approach to provide cognitive scaffold. With the availability of novel structural note-taking tools, such as NoteStruct [32], which organize note-taking processes into three phases, we need to verify how such strategies may help (1) mitigate distraction caused by note-taking during video-based learning, and (2) prompt learners to reflect on the course material and engage learners with the learning content.

Online video-based learners also risk negative impacts due to learning in isolation. The sense of learning with peers in a community can similarly decline given the lack of peer interactions, which raises the need of seeing the traces of other learners, as well as sharing and exchanging thoughts with others as what's readily available and supported in traditional classrooms [2, 47] Upon the basis of NoteStruct, we investigate the effects of structured note-taking in a social learning context, which enables learners to share notes in the form of learning traces, and construct notes with support of these traces made available on a structured note-taking and sharing system. Notesharing is one typical way students provide social support to their peers. As an external cognitive resource [21], notes can mediate rich information of learners' cognitive processing on specific course content (e.g., attention, thinking, reflection etc.), which could all be meaningful information to share, adding useful cues to the rest of the learning community. Thus, in the social learning context, we have designed and prototyped another structured note-taking tool, NoteCoStruct. Similar to NoteStruct, NoteCoStruct supports three-phase structured note-taking. Additionally, in each phase, NoteCoStruct displays learning traces left by previous learners of the video in the form of highlights, comments, or finalized full notes. The sharing is unidirectional - on the one hand, learners can access traces left by peers learned before them, and on the other hand, their products

Proc. ACM Hum.-Comput. Interact., Vol. 6, No. GROUP, Article 21. Publication date: January 2022. 
of each phase of NoteCoStruct can serve as learning traces and social annotations for subsequent learners, which are potentially rich sources for social scaffold.

In an online experiment, we systematically evaluated NoteStruct [32] and NoteCoStruct, investigating the effects of structured note-taking on learners' experiences and note-taking behaviors in video learning. With a mixture of self-report, activity log, and content analysis, our results showed that taking notes with structured tools systematically improved learners' cognitive engagement, lowered distraction, and improved notes completeness and usefulness in both individual and social learning scenarios. However, the social effects of how sharing notes impact learners are nuanced and inconclusive.

The contribution of the paper is twofold. First, we prototyped a structured note-taking system, NoteCoStruct to enable sharing of peer note-taking traces in video-based learning, and to provide social scaffolds to learners. Also, we evaluated the effects of structured note-taking in both individual and social learning scenarios to gain in-depth understanding on how structured note-taking systems affect video-based learning.

\section{BACKGROUND}

\subsection{From note-taking to structured note-taking}

Note-taking is a ubiquitous practice for traditional in-classroom learners, serving as their primary strategy to learn critical course content [16]. Students have traditionally taken notes in a longhand approach, but the prevalence of digital devices fueled their transition to digital note-taking methods [44]. In online learning scenarios, note-taking is also regarded as an important learning strategy and contributes to students' learning performance [35].

Empirical studies have proposed two main reasons as to the significance of note-taking for learning $[12,15,21,27]$. On the one hand, from the perspective of encoding, taking notes enhances the acquisition of information, serving as a cognitive aid to help learners identify and organize important information. On the other hand, from the perspective of external storage, taking notes benefits learning by assisting learners with recalling the lecture content at a later stage. As byproducts of these two key functions, note-taking allows for active engagement during lectures [5], and effective note takers are rewarded with better academic performance [49]. Although these positive correlations have been widely acknowledged, researchers have stressed that an active learning process that involves information comprehension, selection, and production is required, rather than passively transcribing what is heard [43]. In other words, ensuring quality note-taking is critical to learners' performance [27].

High-quality notes are expected to be complete, elaborative, and organized [22]. Based on these three key characteristics, researchers have developed numerous methods to evaluate the quality of learners' notes, from both qualitative and quantitative perspectives. Qualitative measures mostly rely on manual ratings or scoring $[41,54]$, and quantitative measures use statistics of note content such as number of idea units recorded, number of details, number of words, clarity, legibility, sequencing, and accuracy of notes [38, 42].

However, high-quality note-taking is deemed challenging for many learners, especially during fast-paced lectures. Taking notes while listening and processing content simultaneously puts students under severe time pressure, and costs attention needed for processing rapid and dense lecture presentations, which may hinder learners' comprehension [32]. Compared with traditional paper-based note-taking, digital note-taking introduces more affordances to enable fast recording, but meanwhile introduces additional challenges. For example, video-based learners tend to directly transcribe lectures verbatim as their notes, which requires a relatively shallow cognitive process $[18,33,50]$. 
The initial design and evaluation of NoteStruct [32] showed the potential of structured notetaking to scaffold video-based learners' note-taking processes, lowering the barrier of taking notes while watching videos, and prevent several drawbacks of digital note-taking. The general design of NoteStruct walks learners through a step-by-step process that breaks holistic note-taking activities into several less demanding phases, helping to reduce interference of note-taking while watching lecture videos and prevent learners from taking verbatim or shallow notes [32].

In the current work, we conduct an experiment using the newest version of NoteStruct which employs a three-phase note-taking structure: transcript highlighting, commenting, and notes finalizing, and further studied the effects of structured note-taking from more perspectives.

\subsection{Distraction and engagement in online video-based learning}

To ensure learning effectiveness and quality, learners need to direct their attention resources to the learning content and actively avoid external and internal distractions [46]. Yet, maintaining longterm attention and avoiding mind-wandering is challenging for self-paced video-based learners [46]. Computer-related multitasking (e.g., web searching and emailing) and learning-related multitasking (e.g., note-taking) may distract learners and cause them to miss important points in lectures, which may lead to poor learning performance [1,39]. Additionally, as videos are pre-recorded, instructors can hardly employ strategies of real-time attention guidance [17], leaving learners to confront distraction alone. Due to lack of interactivity with learning materials, individual learners can easily become disengaged [25] and unable to reflect on what they have learned [48].

It is evident that passively watching online videos is not sufficient for learning and constructing learning concepts [28]. Thus, to support individual learners actively interacting with the online video learning content, several approaches have been proposed. For example, Shin et. al. designed in-video prompts to facilitate learners to reflect on course materials [48]. Guo et. al. provided several practical guidelines by suggesting instructors putting efforts into making online videos, such as making courses into short chunks or adding informal content in the video [14]. These approaches may introduce extra work to instructors (e.g., designing appropriate in-video prompts) or require instructors to redesign the presentation of videos posted in the past.

In this study, we suggest the potential of using structured note-taking systems to scaffold videobased learners avoid distraction and cognitively engage in learning while not introducing extra burden to instructors and course designers.

\subsection{Bringing social annotations into online learning}

Social annotations, which are digital traces such as notes, highlights, bookmarks left by online users, have been widely utilized in social computing systems. Social annotations have been found effective in supporting information consumption (e.g., reading online news [29], web searching [34], knowledge sharing in online communities such as Wikipedia [24] and sensemaking [36]. The benefits of social annotations lie in supporting individual decision-making when deciding what to or not to consume [51], and providing social contexts to enhance engagement while consuming online content [29].

In more formal education settings, to reduce individual learners' feeling of isolation while watching online videos, Liao et. al. proposed aggregating course comments, as a type of social annotations, from prior learners to create virtual classmates [31]. In MOOCs where large-scale online learners actively consume learning materials individually and leave digital traces while learning, the social annotations left by online learners could be rich sources to facilitate learning. Thereby, in this study, we curate social annotations from online learners and investigated whether it is an effective way to mitigate learners' feeling of isolation, enhance their sense of learning community, and support learning activities while watching lecture videos alone. 
Understanding the Effects of Structured Note-taking Systems for Video-based Learners in

Individual and Social Learning Contexts

\subsection{Research questions}

To understand the effects of structured note-taking when learning individually versus with the aid of learning traces, we pose the following research questions:

$R Q 1$. On the individual level, how does structured note-taking affect students' learning experience? RQ2. On the social level, how does displaying social annotations affect students' learning experience?

\section{SYSTEM DESIGN}

\subsection{The design of structured note-taking systems: NoteStruct and NoteCoStruct}

In this section, we describe the general system design of structured note-taking systems for individual learning, NoteStruct, and how it is extended to the social version, NoteCoStruct. Both structured note-taking systems employ a three-phase structure. Table 1, Table 2, and Table 3 shows the interface design of each phase in NoteStruct and NoteCoStruct systems.

3.1.1 Phase 1: Video-watching and Transcript Highlighting. In this first phase, the learners watch the lecture video and are provided the corresponding time-anchored transcript. Learners are prompted to highlight the parts of the transcript that they think are important. As one of the most widespread learning strategies, highlighting helps students to pay attention to important information [30,52]. Since highlighting is a lightweight task and requires much less time and effort than writing down full notes, learners may be less distracted from the ongoing lecture video and feel less demanding. In NoteCoStruct, we visualized and presented past learners' highlights by putting a green vertical bar on the left side of the highlighted parts of the transcript, where darker green means more learners have highlighted.

3.1.2 Phase 2: Commenting on Highlighted Parts. One of the major functions of highlighting learning materials is to transfer the information into working memory for further process [52]. In the second phase, learners are asked to comment on their previously highlighted parts one by one to stimulate learners to retrieve related information from memory and to trigger learners' deeper cognitive processing of the learning material. Instead of merely transcribing the presented information, which is not an effective learning strategy [33], structured note-taking systems encourage learners to provide supporting details, examples, and their own understanding. In other words, inspired by the benefits of retrieving information from memory and reflection $[9,53]$, the systems aim to integrate course material reflection and explanation into this phase of note-taking process. In NoteCoStruct, the system displays comments written down by previous learners to provide social scaffold. Such comments are usually short, fragmented, and thus we don't expect learners to feel overwhelmed to read and absorb such content. Note that in both structured systems, learners are not forced to comment on every highlight and they can skip if they would like to.

3.1.3 Phase 3: Notes Finalizing. Preliminary notes are automatically generated based on learners' highlights and comments in the previous two phases, and it can serve as a starting point for note finalizing. In the third phase, learners will be able to freely edit the preliminary notes (e.g., adding additional information, deleting unnecessary or redundant parts, or changing the layouts). In NoteCoStruct, peers' completed notes are also displayed in this phase for learners' reference.

\subsection{Prototyping baseline systems of holistic note-taking and note-sharing}

To investigate the effectiveness of learning with structured note-taking systems, we designed baseline note-taking systems in both individual and social contexts in order to compare the structured note-taking systems to them in a controlled online study. The baseline systems only have one phase and support basic video-watching and digital note-taking functions. Additionally, in the baseline 


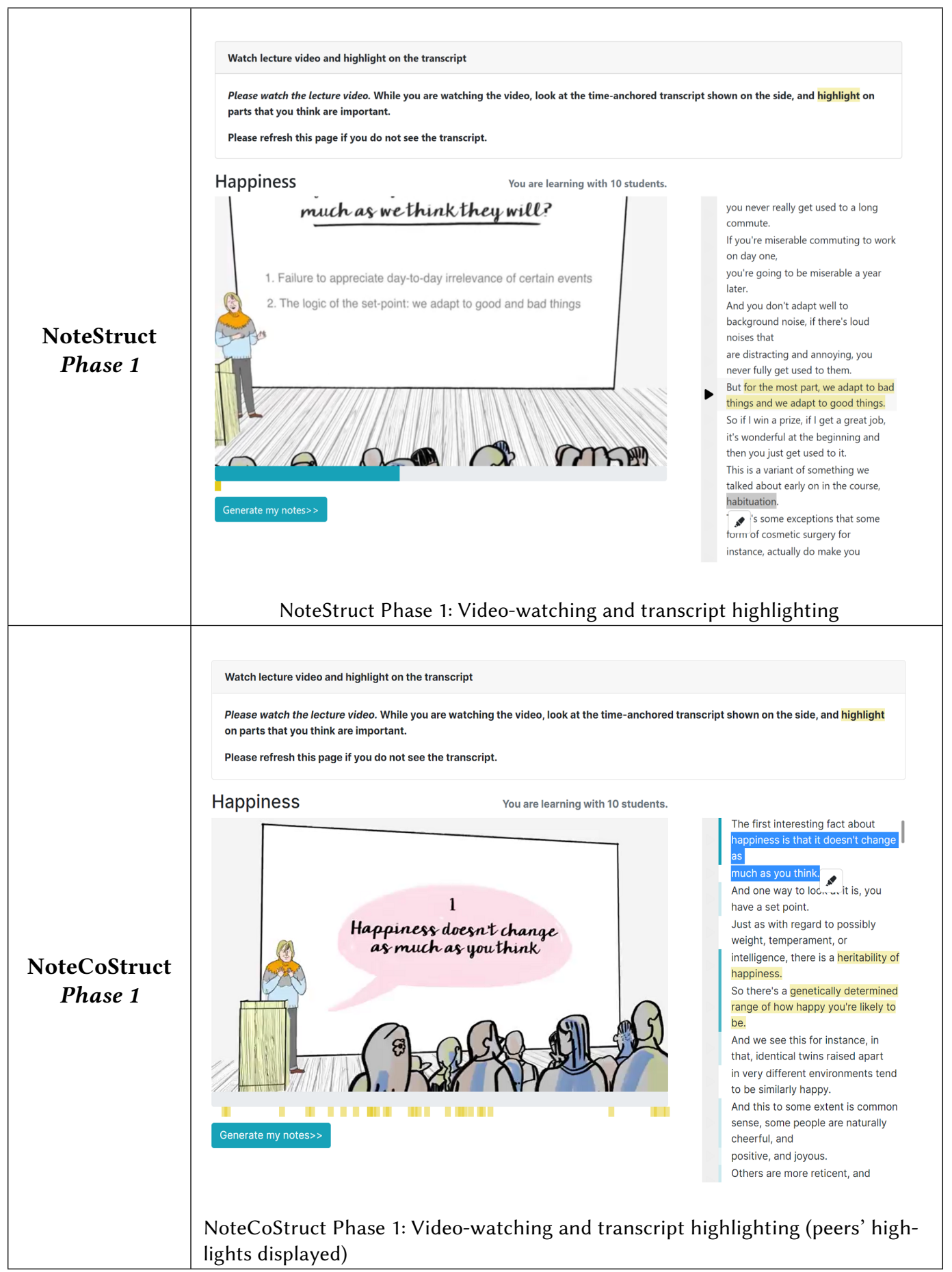

Table 1. Phase 1 of NoteStruct and NoteCoStruct 
Understanding the Effects of Structured Note-taking Systems for Video-based Learners in

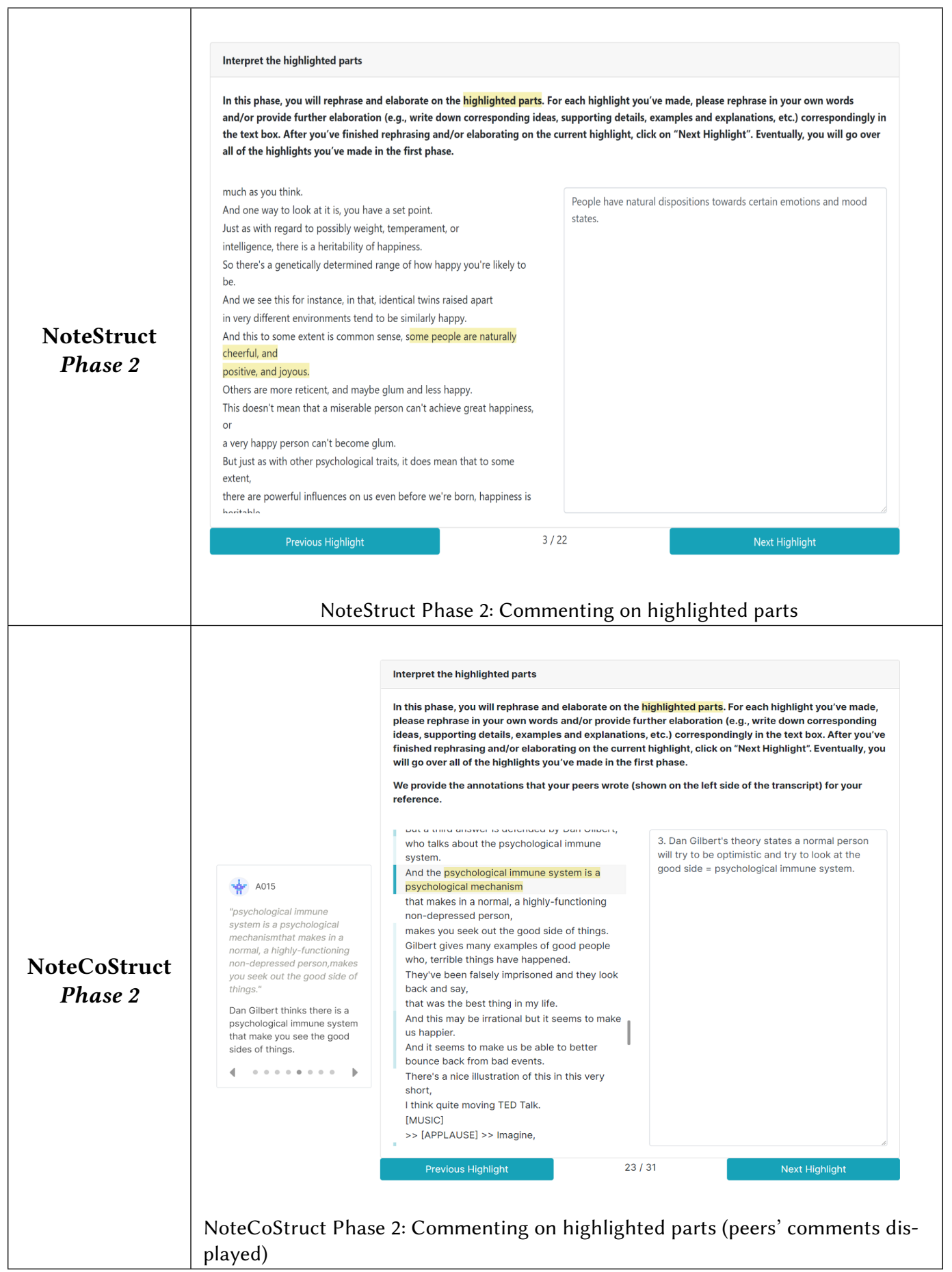

Table 2. Phase 2 of NoteStruct and NoteCoStruct 


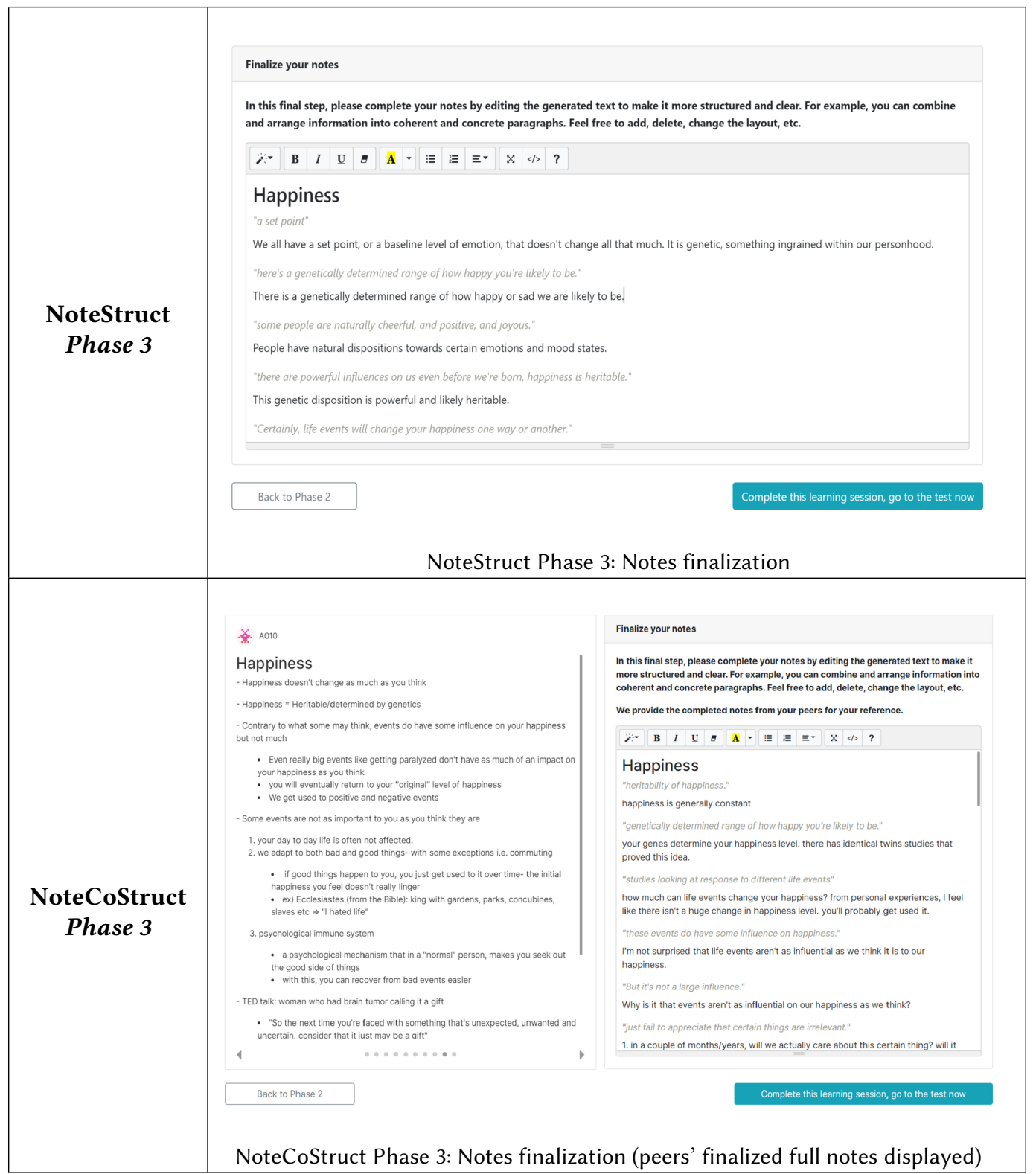

Table 3. Phase 3 of NoteStruct and NoteCoStruct

system designed for the social learning context, peers' holistic notes are shown to learners. This note-sharing feature in the baseline system is designed to be comparable to common practices of sharing only peers' completed notes without detailed learning traces (i.e., highlights and comments), and online discussion forums can theoretically support this basic note-sharing function as well. Table 4 shows the interfaces of Individual Baseline system and Social Baseline system. 
Understanding the Effects of Structured Note-taking Systems for Video-based Learners in

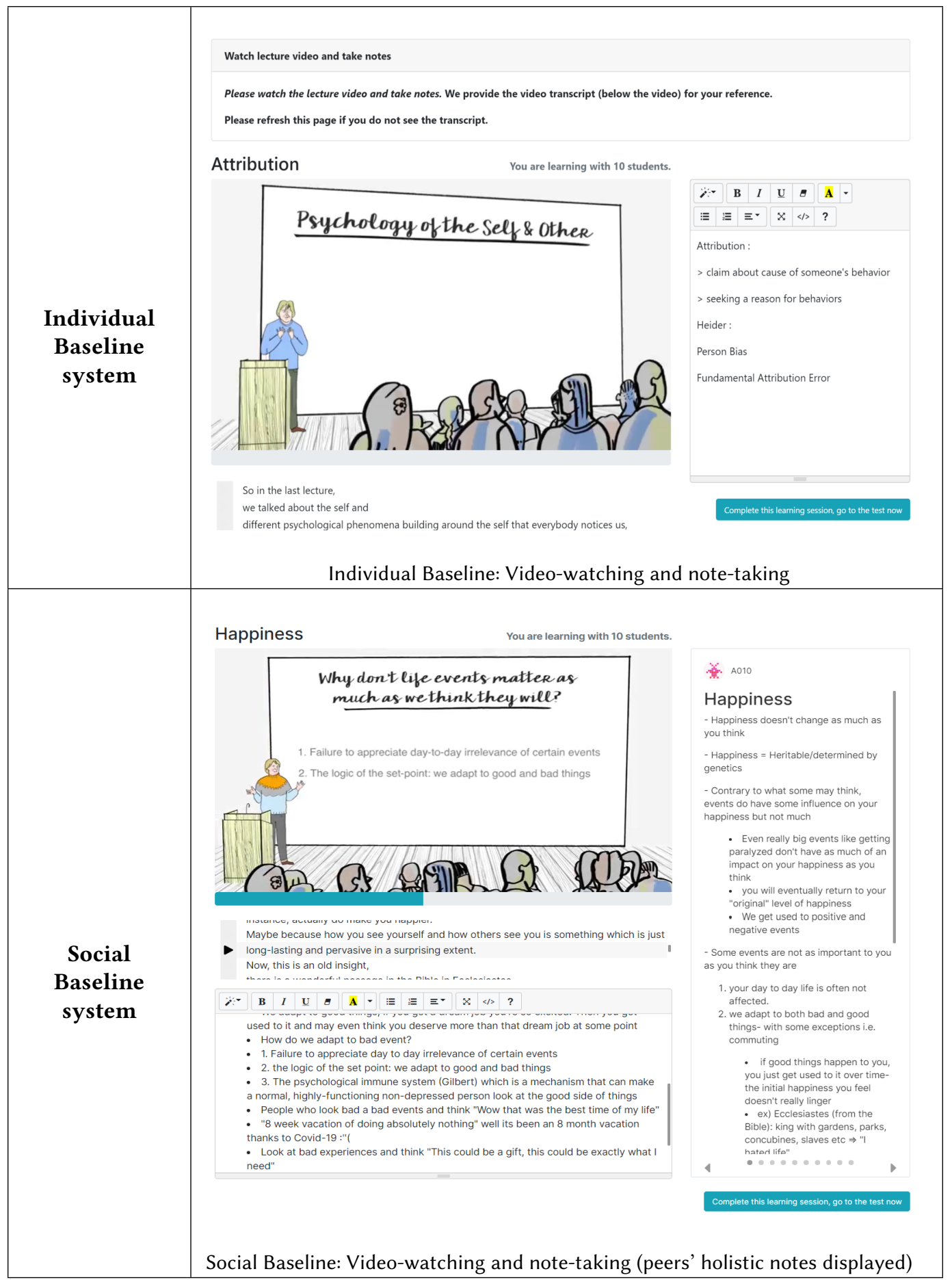

Table 4. Baseline systems 


\begin{tabular}{|c|l|l|}
\hline Baseline & \multicolumn{1}{|c|}{ Individual } & \multicolumn{1}{|c|}{ Social } \\
\hline Structured & NoteStruct & $\begin{array}{l}\text { Video-watching \& note-taking with } \\
\text { peers' notes }\end{array}$ \\
\hline
\end{tabular}

Table 5. System used in each experimental condition

\section{METHOD}

We conducted a $2 \times 2$ factorial experiment to understand the effects of structured note-taking in individual and social video learning contexts. The four prototypes described above reflect the four experimental conditions (i.e., two factors, each consisting of two levels) shown in Table 5.

\subsection{Collecting learning traces and notes for sharing}

To collect learning traces and holistic notes which will be shown in NoteCoStruct and the social baseline system, we recruited 11 participants to go through two learning sessions and take notes using NoteStruct. One learning session included a 10-minute lecture video and the other included a 4-minute lecture video, with the order of videos counterbalanced. Both videos are retrieved from university-level psychology classes on Coursera. We excluded notes from one participant who did not perform serious learning and note-taking (specifically, did not play the lecture video), thus only social notes and learning traces from 10 participants were included. In total, 10 pieces of completed notes for each lecture video were collected, and participants generated 220 highlights and 198 comments for the 10-minute lecture, and 120 highlights and 105 comments for the 4-minute lecture.

\subsection{Recruitment and study procedure}

We recruited participants from an online recruitment platform (Prolific ${ }^{1}$ ), social media (Facebook groups and Reddit), and mailing lists of a public university in the United States. All of them are current undergraduate or graduate students living in the United States. Each participant received $\$ 10$ compensation upon completion of the study.

We conducted a $2 \times 2$ between-subject experiment. At the beginning of the study, participants read through the consent form and they were given an overview of the process. Each of them went through two learning sessions (i.e., video-watching and note-taking), a test after each session, and a survey with questions about demographics information and learning experiences at the end of the study. Each learning session either includes the 10-minute video or the 4-minute video provided to the previously recruited 10 note-takers, with the order of videos counterbalanced. They were given a short tutorial of what functions are supported and how to interact with the interface at the beginning of each phase of the learning sessions. Participants were randomly assigned to one of the four conditions to learn and take notes with one of the four systems in both learning sessions.

In total, 52 participants completed the study. We reviewed their data using manual inspections and we removed data generated by 9 participants from the dataset for one of the following reasons: missing data, short survey completion time (i.e., spent less than $2.5 \mathrm{~s}$ on reading and answering each question), and inconsistent or fake responses in the survey. We analyzed data generated by the left 43 participants (18 male, 24 female, 1 agender). Among them, 11 were assigned to Individual Baseline condition and used the Individual Baseline system, 11 were assigned to Social Baseline condition and used the Social Baseline system, 7 were assigned to the Individual Structured condition and

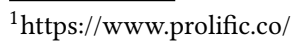


Understanding the Effects of Structured Note-taking Systems for Video-based Learners in

Individual and Social Learning Contexts

used NoteStruct system, and 14 were assigned to the Social Structured condition and used the NoteCoStruct system. Participants took 56 minutes to complete the study on average.

\subsection{Measurement}

4.3.1 Survey and test. In this study, we collected participants' responses to survey items which were answered after they experienced two learning sessions with one of the four systems. All survey items were presented in 7-point Likert scale. In the survey, we asked participants about their learning and note-taking experience, specifically:

- To verify whether the three-phase structured note-taking design caused less distraction from the current learning activity, we measured participants' perceived level of distraction caused by note-taking while watching online videos (e.g., "I can concentrate in classes when taking notes") using items adapted from [26].

- Literature shows that cognitive engagement is an important factor affecting learning outcome [7]. Using items adopted from [6], we measured participants' cognitive engagement (e.g., "When I am taking the courses with this tool, I am absorbed by course content").

- Lacking a sense of learning community could lead to negative effects such as course dissatisfaction and low retention rate [20]. Using items adopted from [40], we measured participants' sense of learning community (e.g., "I feel like a member of the community formed by the learners of these two classes").

- It is widely believed that reviewing notes is beneficial, yet learners often take incomplete notes that are limited for later use [21]. We measured notes usefulness by asking learners' perceived learning gain of reviewing notes using one item ("I have more learning gain by reviewing my notes with this tool").

To measure learning outcome, we designed one test for each of the lecture videos. Both tests include a content summarization task (i.e., "Please summarize the lecture content in a paragraph (in less than 200 words)"), and two open-ended conceptual questions (e.g., "What is attribution theory? Elaborate with one example."), which are closely related to the key concepts mentioned in the videos. The raw scores of the summarization tasks are given by coding and counting the number of knowledge units mentioned in each participants' responses, and after grading all the responses, we normalized the score by dividing each participants' raw score by the highest raw score of that test, times 10 . The conceptual questions are graded based on whether the concepts are explained correctly and whether the examples are reasonable. Each participants' final score was calculated by summing the scores of all questions in the two tests. The full score is 55 . Initially, two graders graded the same set of test responses ( $20 \%$ of all responses), reached Kappa $=0.77$ and resolved the differences, and then moved on to grade the rest.

4.3.2 Activity log. We collected participants' log data from the systems to analyze their behavior in video watching. Specifically, with timestamps of video start, pause and restart, and video end, we calculated each participants' video watching rate for each of the two videos by computing $\frac{\text { duration of video-watching }}{\text { time length of the video }}$, and then derived participants' overall watching rate by calculating the average of the videos. Video watching rate larger than 1 means that participants rewatched some part of the video.

Although video rewatching allows participants to set their own pace for learning [37], research showed that it may cause frustration and increase extraneous load by causing a disruption $[8,11]$ Moreover, rewatching could be a learning strategy that learners intentionally used to reiterate points, to complete note-taking, or to remain focused [11], which implies that a high video-watching rate (when video-watching rate $>1$ ) could be a signal of students being distracted during learning. 
4.3.3 Notes content. Notes, as external storage and products of learning, may reveal learners' depth of knowledge processing. We pre-processed learners' notes in order to perform content analysis.

Text similarity. To investigate how much are learners affected by social annotations generated by peers, we calculated the text similarity between full notes generated by participants with the aggregation of 10 pieces of pre-collected social notes. We adopted token-based Jaccard similarity, which reflects the proportion of similar tokens in two text pieces.

Since each participant went over two learning sessions (video-watching and note-taking) and generated two pieces of full notes, we calculated the average similarity score of the two and used it in data analysis.

Comments/notes content categorization. To investigate how social annotations could affect the type of content generated from structured note-taking, especially the comments generated in phase 2 of the structured systems (which will be integrated into full notes in phase 3), we proposed to extend an existing analytical framework for general learning behaviors to categorize the comments (or notes) content. This analysis is applied to structured note-takers only (i.e., learners using NoteStruct and NoteCoStruct).

We are inspired by the ICAP (Interactive, Constructive, Active, Passive) framework which describes and categorizes learning behaviors into four modes (i.e., passive, active, constructive, and interactive) and posits that by transforming a learner's behavior from the passive to the active, to the constructive, and to the interactive mode of learning, students become increasingly more engaged with learning activities, also leading to greater learning gain [10]. As notes produced through note-taking activities can externalize learners' cognitive and learning states and may contain rich information to be studied, we adapted the ICAP framework to analyze the content of comments generated in the Phase 2 of structured note-taking systems. Although passive learning may not be observed based on these comments, the rest of the modes were all observed in Phase 2 as defined in Table 6.

This adapted framework aims to capture meaningful commenting behaviors in both individual level and social level structured note-taking. From the aspect of individual learning, similar to the prediction of ICAP, we predict that learners are more cognitively engaged as learners take active notes to constructive notes to interactive notes. As for note sharing and social learning, active notes that were directly copied and pasted from transcripts barely provide extra information to peers. In contrast, constructive notes tend to contain worked-out examples or partially processed knowledge that could potentially stimulate peers' processing of the content, and interactive notes have the potential to enhance peers' engagement and sense of learning community, hence such comments could be deemed to be more valuable social support to subsequent learners. Thus, learners who use social note-taking systems might have the intention to effectively support peers and take more constructive and interactive notes, which are beneficial learning strategies for themselves as well

After constructing the coding scheme based on initial observations, two independent coders coded a set of comments based on the adapted framework and categorized each comment into one of the four types: active, constructive, interactive, and other. We moved on to code the rest after correlation $=0.80$ was reached. After all of the comments were coded, for each learner, we calculated the percentage of active comments by evaluating $\frac{\text { number of active comments generated by the learner }}{\text { number of all comments generated by the learner }}$. Similarly, we calculated the percentage of constructive comments, the percentage of interactive comments, and the percentage of other comments for each learner.

Notes word count. As a factor of notes completeness, we also considered word count of each piece of notes as a measurement. We assume that notes with more word counts are likely to be 


\begin{tabular}{|l|l|}
\hline The original ICAP Framework & $\begin{array}{l}\text { Adapted framework for analyz- } \\
\text { ing comments }\end{array}$ \\
\hline $\begin{array}{l}\text { PASSIVE RECEIVING } \\
\text { e.g., Listening to a lecture without do- } \\
\text { ing anything else. }\end{array}$ & $\begin{array}{l}\text { N/A } \\
\text { (Cannot be detected from notes con- } \\
\text { tent.) }\end{array}$ \\
\hline $\begin{array}{l}\text { ACTIVE MANIPULATING } \\
\text { e.g., Repeating or rehearsing; Taking } \\
\text { verbatim notes. }\end{array}$ & $\begin{array}{l}\text { ACTIVE NOTES: } \\
\text { Taking verbatim notes such as copy- } \\
\text { paste and copy-delete; Highlighting } \\
\text { without writing comments.) }\end{array}$ \\
\hline $\begin{array}{l}\text { CONSTRUCTIVE GENERATING } \\
\text { e.g., Reflecting out-loud; Self- } \\
\text { explaining. }\end{array}$ & $\begin{array}{l}\text { CONSTRUCTIVE NOTES: } \\
\text { Self-explaining; Rephrasing; Elaborat- } \\
\text { ing with new ideas. }\end{array}$ \\
\hline $\begin{array}{l}\text { INTERACTIVE DIALOGUING } \\
\text { e.g., Debating } \text { or arguing. }\end{array}$ & $\begin{array}{l}\text { INTERACTIVE NOTES: } \\
\text { Expressing personal feelings; Express- } \\
\text { ing agreement or disagreement; Ask- } \\
\text { ing or answering questions. }\end{array}$ \\
\hline $\begin{array}{l}\text { N/A } \\
\text { OTHER NOTES: } \\
\text { Random notes or unrelated text. }\end{array}$ \\
\hline
\end{tabular}

Table 6. Adapted ICAP framework for analyzing comments in structured note-taking

more complete. Again, since each participant generated two pieces of full notes, we calculated the average word count of the two.

\subsection{Hypotheses}

Given that structured note-taking systems divide the note-taking process into a series of lessdemanding phases, separate the lecture video-watching and the major workload of note-taking into different phases, and motivate learners to reflect and elaborate, we proposed hypotheses $\mathrm{H} 1$ and $\mathrm{H} 2$ :

H1. With structured note-taking systems, participants are less likely to be distracted and more cognitively engaged with the learning materials than without structured note-taking system support.

H2. With structured note-taking systems, participants can take more complete and useful notes than without structured note-taking system support.

Since social annotations could offer various social and learning benefits, we hypothesize that sharing learning traces and notes among peer learners in education settings have similar effects and proposed $\mathrm{H} 3$ :

H3. With social note-taking systems which present annotations shared by peers, participants can perceive a stronger sense of learning community, and take more similar notes to social notes that they were exposed to.

Verbatim notes (i.e., active notes in our adapted ICAP framework) do not include any extra information being mentioned in the videos, therefore in the social context when notes are shared, verbatim notes are futile for subsequent learners to view. Thus, we proposed H4:

H4. With social note-taking systems which present annotations shared by peers, participants tend to take more constructive and interactive notes.

Finally, based on potential benefits of cognitive engagement and sense of learning community with note sharing, which may contribute to learning gain, we proposed H5: 
H5. Participants using structured systems can achieve higher learning outcomes than those using baseline systems, and participants using social systems with peers' annotations can achieve higher learning outcomes than those using individual systems.

\section{RESULTS}

\subsection{Distraction and cognitive engagement}

Two-way ANOVA showed that participants using structured note-taking systems perceived significantly lower distraction caused by note-taking $(F[1,39]=6.20, p<0.05$; baseline systems: $M=2.41$, $\mathrm{SD}=1.20$; structured systems: $\mathrm{M}=1.45, \mathrm{SD}=1.11$ ) (see Fig. 1).

The average video-watching rate of learners using structured systems is $1.05(\mathrm{SD}=0.16)$, which is significantly less than 1.23 ( $\mathrm{SD}=0.31$ ), the average watching rate of learners using baseline systems $(\mathrm{F}[1,39]=4.86, \mathrm{p}<0.05)$. Since both average rates are larger than 1 , it suggests that learners using structured systems spent less time rewatching the video, which implies that they are cognitively more efficient and less distracted in processing the videos.

We examined how structured note-taking affects learners' level of cognitive engagement with learning materials. Two-way ANOVA revealed that learners using structured note-taking systems reported significantly higher levels of cognitive engagement $(\mathrm{F}[1,39]=5.82, \mathrm{p}<0.05$; baseline systems: $\mathrm{M}=5.39, \mathrm{SD}=1.17$, structured systems: $\mathrm{M}=6.22, \mathrm{SD}=0.91$ ), showed in Fig. 1 .

Altogether, these results support H1.

\subsection{Social effects}

Two-way ANOVA did not show evidence of note-taking using social systems (either structured or baseline) could enhance the sense of learning community $(\mathrm{F}[1,39]=0.16, \mathrm{p}=0.69$; individual systems: $\mathrm{M}=4.87 ; \mathrm{SD}=1.58$; social systems: $\mathrm{M}=4.89 ; \mathrm{SD}=1.60)$. Surprisingly, we found that learners using structured systems perceived a higher level of sense of learning community than using baseline systems $(\mathrm{F}[1,39]=8.63, \mathrm{p}<0.01$; baseline systems: $\mathrm{M}=4.21, \mathrm{SD}=1.57$; structured systems: $\mathrm{M}=5.59, \mathrm{SD}=1.26$ ). Post-hoc contrast analysis showed that learners using NoteCoStruct reported a significantly higher sense of learning community than learners using the Social Baseline system $(\mathrm{F}[1,39]=9.12, \mathrm{p}<0.01$; NoteCoStruct: $\mathrm{M}=5.67, \mathrm{SD}=1.25$; Social Baseline: $\mathrm{M}=3.90 ; \mathrm{SD}=1.48$ ) (showed in Fig. 2), while no significant difference in sense of learning community was found between using NoteStruct and the Individual Baseline system.

We then moved on to compare the similarity between learners' notes and pre-collected social notes. Again, two-way ANOVA did not show evidence that final notes generated by participants using social systems are more similar to pre-collected social notes than notes generated by those using individual systems. Yet, we found that learners using structured systems generated significantly more similar notes to social notes, compared to notes generated by baseline systems (F[1, $39]=5.59, \mathrm{p}<0.05$; baseline systems: $\mathrm{M}=0.07, \mathrm{SD}=0.03$; structured systems: $\mathrm{M}=0.11, \mathrm{SD}=0.06)$. Similarly, post-hoc contrast analysis revealed that notes generated by learners using NoteCoStruct are significantly more similar to displayed social notes, compared to notes generated by learners using the Social Baseline system $(\mathrm{F}[1,39]=6.29, \mathrm{p}<0.05$; NoteCoStruct: $\mathrm{M}=0.12, \mathrm{SD}=0.07$; Social Baseline: $\mathrm{M}=0.09$; $\mathrm{SD}=0.03$ ) (see Fig. 3), while no significant difference was observed between notes generated by learners using NoteStruct and notes generated by learners using the Individual Baseline system.

In summary, H3 is not fully supported. However, it is interesting that in the social learning context, learners using NoteCoStruct were affected more by social annotations shared by peers (regarding how much sense of learning community they perceived and how much the content of their notes are affected by displayed social notes) than learning using the Social Baseline system, who can only see peers' holistic notes without more detailed learning traces such as highlights and 
comments. This suggests that as to social learning scenarios, sharing social annotations generated by structured systems is certainly more effective than sharing those generated by baseline systems. The possible explanation will be discussed in the Discussion section.

\subsection{Notes content and quality}

Notes with more word count may imply it is more complete. Two-way ANOVA revealed that learners using structured systems wrote more words in their final notes than learners using baseline systems, showed in Fig. $4(\mathrm{~F}[1,39]=6.07, \mathrm{p}<0.05$; baseline systems: $M=132.30, \mathrm{SD}=65.34$; structured systems: $\mathrm{M}=214.38, \mathrm{SD}=114.49$ ).

Learners using structured note-taking systems also reported higher perceived learning gain by reviewing notes, compared to learners using baseline systems $(F[1,39]=4.09, p=0.05$; baseline systems: $\mathrm{M}=5.09, \mathrm{SD}=1.48$; structured systems: $\mathrm{M}=5.81, \mathrm{SD}=1.29$ ). This suggests that learners consider notes generated by using structured note-taking systems to be more useful.

These results support $\mathrm{H} 2$.

To investigate whether using individual or social systems can affect the content of notes taken by learners, we then compared the type of comments (which will be included in final notes) generated by learners using NoteStruct and NoteCoStruct. When taking notes with NoteStruct, on average, learners wrote $8.95 \%$ of active comments $(\mathrm{SD}=0.11), 83.70 \%$ of constructive comments $(\mathrm{SD}=0.15), 5.14 \%$ of interactive comments $(\mathrm{SD}=0.12)$, and $2.19 \%$ of other comments $(\mathrm{SD}=0.06)$; while learners who took notes with NoteCoStruct wrote $8.19 \%$ of active comments $(\mathrm{SD}=0.11), 84.12 \%$ of constructive comments $(\mathrm{SD}=0.19), 6.60 \%$ of interactive comments $(\mathrm{SD}=0.13)$, and $1.10 \%$ of other comments $(\mathrm{SD}=0.02)$. No significant difference was found between the two condition groups based on one-way ANOVA.

Thus, H4 is not supported.

\subsection{Learning outcome}

Regarding learning outcome, two-way ANOVA result showed no significant difference between learners using structured systems and learners using baseline systems. Also, learners using social note-taking systems did not achieve significantly higher learning outcomes than those using individual note-taking systems. Hence, H5 is not supported.

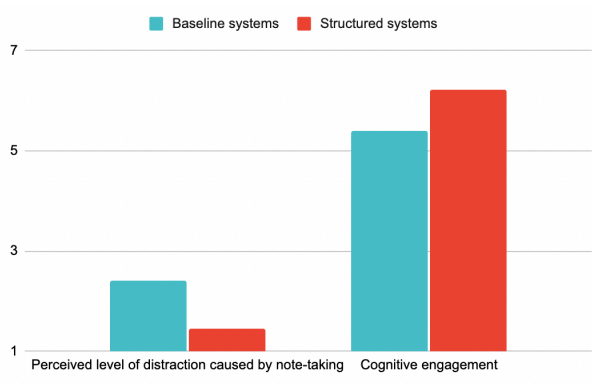

Fig. 1. Comparing distraction and cognitive engagement between learners using structured versus baseline systems.

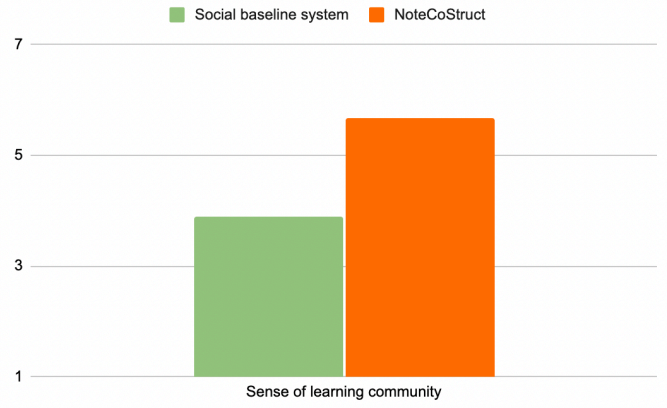

Fig. 2. Comparing perceived sense of learning community between learners using NoteCoStruct and social baseline. 

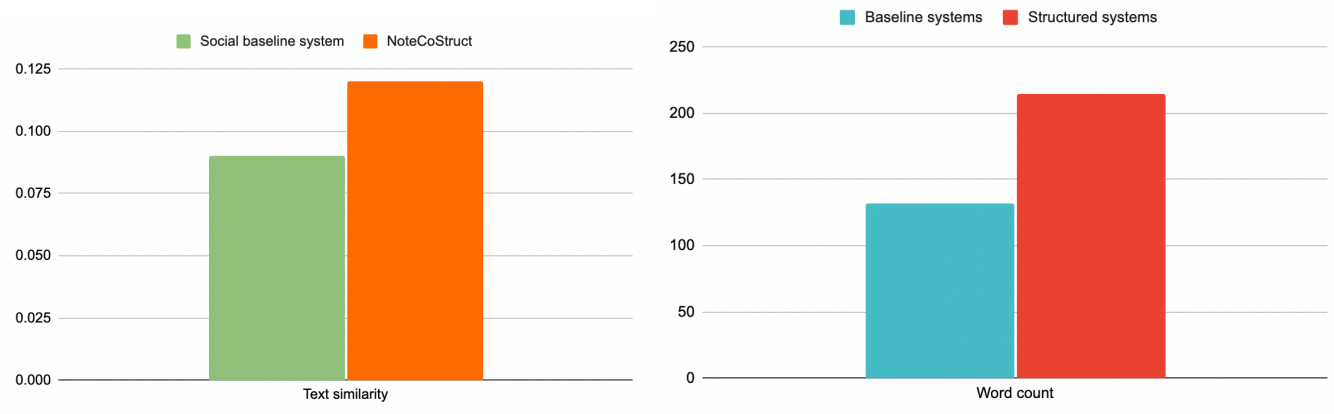

Fig. 3. Comparing text similarity to pre-collected Fig. 4. Word counts of notes between learners using social notes between learners using NoteCoStruct structured versus baseline systems.

and social baseline.

\section{DISCUSSION}

In summary, our results suggest that structured note-taking systems successfully provided cognitive scaffold in learners' learning process and helped learners to take longer and better notes for future use. However, the social effects introduced by social systems (NoteCoStruct and Social Baseline) is more complex. We will discuss key results in this section.

\subsection{The cognitive effects of structuring note-taking process in video-based learning}

Studies revealed that while video-based learners are expected to sustain attention in watching and absorbing video content, they may be distracted by nuanced activities, events and even note-taking itself $[1,39]$. As posited in H1, with structured note-taking systems, we expected learners to be less distracted and more cognitively engaged in the learning processes because the phase-by-phase workflow is basically designed to guide them to distribute note-taking into less demanding subtasks which avoid multitasking. In addition, in structured systems, learners were prompted to reflect on the key points they highlighted, which first requires learners to retrieve related course information from their memory, then deeply process the information to generate their elaborations, explanations, or examples in their own words. Such practices could have motivated learners to perform active thinking throughout the note-taking process. Our analysis results based on survey responses and activity log consistently support $\mathrm{H} 1$.

As a consequence of fostering careful and in-depth knowledge processing, structured note-taking systems were also found to help learners generate more complete notes which could better facilitate students to recall information when using notes to review [21], which is in consistence with the prediction of $\mathrm{H} 2$.

\subsection{The social effects of presenting peers' learning traces versus holistic notes}

NoteCoStruct system and Social Baseline system, designed for the social learning context, both present social annotations shared by past learners, but in two different styles: learning traces including highlights, comments, and finalized full notes, or peers' holistic full notes. Implicated by the effects of social annotations in various contexts (e.g., [29, 51], we posed H3, which hypothesized that the existence of social annotations can enhance learners' sense of community and may cause learners to take similar notes. Nevertheless, comparing survey responses and notes content between learners using social systems versus individual systems, our results did not show evidence of social effects associated with taking notes with social annotations. What we found was that in the 
social learning context, compared to learners using the Social Baseline system, learners using NoteCoStruct perceived a stronger sense of learning community, and their notes were also more similar to peers' notes they were shown in the system.

To explain and extend the surprising finding, we need to go back to the original designs of the two social note-taking systems. In the Social Baseline system, only peers' holistic notes, which tend to be long and more cognitively demanding to read and reason, are displayed to subsequent learners. Although the amount of information included in holistic notes is similar to what was provided to learners using NoteCoStruct (as the two systems displayed the same 10 pieces of pre-collected notes), thus still have the potential to provide equivalent academic support, the big chunks of unsegmented notes can become extra burdens and learners may be unable and/or reluctant to read them thoroughly given their limited cognitive and motivational resources. On the contrary, detailed learning traces presented in multiple forms (highlights, short comments, along with finalized full notes) shown to learners using NoteCoStruct are small-packaged information for providing immediate support to learn specific parts of learning material, thus more likely to be proactively digested by learners. Learning traces can also show peers' detailed procedures of knowledge processing, which could provide social transparency of behaviors [13], enhancing peers' social presence and make subsequent learners feel being accompanied and supported when they view social annotations presented in this way. Consequently, it's actually reasonable to see NoteCoStruct learners acquiring a stronger sense of learning community, and the contents of their notes being affected by the notes displayed to them.

The observed differential social effects caused by displaying peers' learning traces versus holistic notes suggest that the presentation style of social annotations matters. When designing video-based learning systems or learning communities for online learners, mindlessly sharing random forms of annotations produced by peers may not effectively eliminate learners' feeling of isolation or provide social scaffold. Rather, the way of displaying the content of social annotations needs to be carefully structured to coach learners to consume the content systematically and to direct viewers' attention to specific important points. System designers could consider using techniques such as visualization for highlighting key phrases or sentences to trigger selective attention (referring to phase 1 of NoteCoStruct) or splitting holistic text into smaller segments (referring to phase 2 of NoteCoStruct) to motivate learners to digest the content being shared such that the shared annotations could provide effective social support.

\subsection{Learning outcome}

Contradict to H5, we did not find the evidence that either structured systems or social systems could improve learners' learning outcome. One possible explanation is that since participants take tests right after they finished the learning sessions and thus we can only measure short-term learning outcome, the potential advantages of structured note-taking systems (such as information retrieval triggered in phase 2, which may positively affect knowledge retention and long term learning performance), cannot be reflected in the measure. However, structured systems appear to foster productive learning behaviors (e.g., taking more complete and useful notes for later use), so future work can further investigate whether taking notes with structured systems can be beneficial to long-term learning gain.

\subsection{Limitations and future work}

The current study has several limitations. First, opposed to H4, we did not detect differences in respect to the percentage of active, constructive, and interactive comments generated by learners using NoteCoStruct system and the Social Baseline system using our notes content analysis framework, possibly due to the small sample size and the limited sets of videos utilized in this small-scale 
study. Experiments with a larger sample size and a larger set of video stimuli may complement this study and further verify whether learning in a social or individual context could induce learners to take different types of notes. Second, in this study, we investigated the social effects caused by displaying social annotations to learners. Nevertheless, we may not always assume that taking notes similar to peers' notes being shared is a positive effect, and even the benefit of consuming social annotation could be questionable under some circumstances. In actual learning communities which may consist of hundreds of thousands of peer learners, if not more, it is possible that some annotations generated by past learners could be spamming or even providing harmful information. Thus, future work can study how to perform quality control on notes being shared and avoid negative effects caused by sharing social annotations before deploying the structured note-taking systems into video-based learning platforms and communities. Furthermore, future work can also explore the effect of enabling instructors to access learners' annotations as a way to collect learners' feedback for adapting their instructions. As instructors' lecture contents and instructional designs can become more flexible given the availability of structured note-taking support to learners, it merits future research to investigate how instructional and note-taking activities may co-evolve.

\section{CONCLUSION}

In this paper, extended from a structured note-taking system designed for individual-based video learning, we prototyped NoteCoStruct, a structured note-taking system powered by peers' social annotations for online video-based learners. We evaluated the effects of both structured systems from the perspectives of cognitive scaffold (support in the form of structured note-taking workflow) and social scaffold (support in the form of sharing peers' notes and learning traces). With an online controlled experiment comparing the structured systems to baseline systems, we found that on the individual level, learners using structured systems were less distracted when learning, more cognitively engaged with the course materials, and were able to take better notes for later use. On the social level, learners using NoteCoStruct perceived a stronger sense of learning community, and their notes are affected more by social annotations presented to them. Based on the results, we confirm the values of structured note-taking for online video-based learning (e.g., MOOCs) in both individual and social learning settings, which can inform the design, iteration, and adoption of digital note-taking tools for video learners.

\section{ACKNOWLEDGMENTS}

We thank the study participants for their time and effort and anonymous reviewers for their insightful feedback on the paper. The work is supported in part by UC Davis through Hao-Chuan Wang's startup grant and the Global Research Experience in Advanced Technologies (GREAT) Program at UC Davis.

\section{REFERENCES}

[1] Ahlam Alghamdi, Aryn C Karpinski, Andrew Lepp, and Jacob Barkley. 2020. Online and face-to-face classroom multitasking and academic performance: Moderated mediation with self-efficacy for self-regulated learning and gender Computers in Human Behavior 102 (2020), 214-222.

[2] Azad Ali and David Smith. 2015. Comparing social isolation effects on students attrition in online versus face-to-face courses in computer literacy. Issues in Informing Science and Information Technology 12, 1 (2015), 11-20.

[3] Aaron Bauer and Kenneth R. Koedinger. 2006. Pasting and Encoding: Note-Taking in Online Courses. In Proceedings of the 6th IEEE International Conference on Advanced Learning Technologies, ICALT 2006, Kerkrade, The Netherlands, fuly 5-7, 2006. IEEE Computer Society, Los Alamitos, CA, USA, 789-793. https://doi.org/10.1109/ICALT.2006.1652559

[4] Robert A Bjork, John Dunlosky, and Nate Kornell. 2013. Self-regulated learning: Beliefs, techniques, and illusions. Annual review of psychology 64 (2013), 417-444.

[5] Joseph R Boyle. 2001. Enhancing the note taking skills of students with mild disabilities. Intervention in School and Clinic 36, 4 (2001), 221-224. 
Understanding the Effects of Structured Note-taking Systems for Video-based Learners in

Individual and Social Learning Contexts

[6] Gerald F Burch, Nathan A Heller, Jana J Burch, Rusty Freed, and Steve A Steed. 2015. Student engagement: Developing a conceptual framework and survey instrument. fournal of Education for Business 90, 4 (2015), 224-229.

[7] Robert M Carini, George D Kuh, and Stephen P Klein. 2006. Student engagement and student learning: Testing the linkages. Research in higher education 47, 1 (2006), 1-32.

[8] Avner Caspi, Paul Gorsky, and Meira Privman. 2005. Viewing comprehension: Students' learning preferences and strategies when studying from video. Instructional Science 33, 1 (2005), 31-47.

[9] Michelene TH Chi, Nicholas De Leeuw, Mei-Hung Chiu, and Christian LaVancher. 1994. Eliciting self-explanations improves understanding. Cognitive science 18, 3 (1994), 439-477.

[10] Michelene TH Chi and Ruth Wylie. 2014. The ICAP framework: Linking cognitive engagement to active learning outcomes. Educational psychologist 49, 4 (2014), 219-243.

[11] Jamie Costley, Mik Fanguy, Chris Lange, and Matthew Baldwin. 2021. The effects of video lecture viewing strategies on cognitive load. Fournal of Computing in Higher Education 33, 1 (2021), 19-38.

[12] Francis J Di Vesta and G Susan Gray. 1972. Listening and note taking. Fournal of educational psychology 63, 1 (1972), 8.

[13] Thomas Erickson and Wendy A Kellogg. 2000. Social translucence: an approach to designing systems that support social processes. ACM transactions on computer-human interaction (TOCHI) 7, 1 (2000), 59-83.

[14] Philip J. Guo, Juho Kim, and Rob Rubin. 2014. How Video Production Affects Student Engagement: An Empirical Study of MOOC Videos. In Proceedings of the First ACM Conference on Learning @ Scale Conference (Atlanta, Georgia, USA) (L@S '14). Association for Computing Machinery, New York, NY, USA, 41-50. https://doi.org/10.1145/2556325.2566239

[15] James Hartley. 1983. Note-taking research: Resetting the scoreboard. Bulletin of the British Psychological Society 36 (1983), 13-14.

[16] James Hartley and Susan Marshall. 1974. On notes and note-taking. Higher Education Quarterly 28, 2 (1974), $225-235$.

[17] Min Hu and Hao Li. 2017. Student engagement in online learning: A review. In 2017 International Symposium on Educational Technology (ISET). IEEE, IEEE Computer Society, Los Alamitos, CA, USA, 39-43.

[18] L Brent Igo, Roger Bruning, and Matthew T McCrudden. 2005. Exploring Differences in Students' Copy-and-Paste Decision Making and Processing: A Mixed-Methods Study. Journal of Educational Psychology 97, 1 (2005), 103.

[19] Geoff Isaacs. 1994. Lecturing practices and note-taking purposes. Studies in Higher Education 19, 2 (1994), $203-216$.

[20] Richard D Johnson, Steven Hornik, and Eduardo Salas. 2008. An empirical examination of factors contributing to the creation of successful e-learning environments. International fournal of Human-computer studies 66, 5 (2008), 356-369.

[21] Kenneth A Kiewra. 1989. A review of note-taking: The encoding-storage paradigm and beyond. Educational Psychology Review 1, 2 (1989), 147-172.

[22] Kenneth A Kiewra and Nelson F Dubois. 1998. Learning to learn: Making the transition from student to life-long learner. Allyn and Bacon, Boston.

[23] Adrian Kirkwood and Linda Price. 2005. Learners and learning in the twenty-first century: what do we know about students' attitudes towards and experiences of information and communication technologies that will help us design courses? Studies in higher education 30, 3 (2005), 257-274.

[24] Aniket Kittur, Ed H. Chi, and Bongwon Suh. 2009. What's in Wikipedia?: mapping topics and conflict using socially annotated category structure. In Proceedings of the 27th International Conference on Human Factors in Computing Systems, CHI 2009, Boston, MA, USA, April 4-9, 2009, Dan R. Olsen Jr., Richard B. Arthur, Ken Hinckley, Meredith Ringel Morris, Scott E. Hudson, and Saul Greenberg (Eds.). ACM, New York, NY, USA, 1509-1512.

[25] René F Kizilcec and Sherif Halawa. 2015. Attrition and achievement gaps in online learning. In Proceedings of the Second (2015) ACM Conference on Learning@ Scale. Association for Computing Machinery, New York, NY, USA, 57-66.

[26] Melina Klepsch, Florian Schmitz, and Tina Seufert. 2017. Development and validation of two instruments measuring intrinsic, extraneous, and germane cognitive load. Frontiers in psychology 8 (2017), 1997.

[27] Keiichi Kobayashi. 2005. What limits the encoding effect of note-taking? A meta-analytic examination. Contemporary Educational Psychology 30, 2 (2005), 242-262.

[28] Kenneth R Koedinger, Jihee Kim, Julianna Zhuxin Jia, Elizabeth A McLaughlin, and Norman L Bier. 2015. Learning is not a spectator sport: Doing is better than watching for learning from a MOOC. In Proceedings of the second (2015) ACM conference on learning@ scale. Association for Computing Machinery, New York, NY, USA, 111-120.

[29] Chinmay Kulkarni and Ed Chi. 2013. All the news that's fit to read: a study of social annotations for news reading. In 2013 ACM SIGCHI Conference on Human Factors in Computing Systems, CHI '13, Paris, France, April 27 - May 2, 2013, Wendy E. Mackay, Stephen A. Brewster, and Susanne Bødker (Eds.). Association for Computing Machinery, New York, NY, USA, 2407-2416.

[30] Detlev Leutner, Claudia Leopold, and Viola den Elzen-Rump. 2007. Self-regulated learning with a text-highlighting strategy. Zeitschrift für Psychologie/fournal of Psychology 215, 3 (2007), 174-182.

[31] Meng-Yun Liao, Ching-Ying Sung, Hao-Chuan Wang, Wen-Chieh Lin, and Fu-Yin Cherng. 2019. Embodying historical learners' messages as learning companions in a VR classroom. In Extended Abstracts of the 2019 CHI Conference on Human Factors in Computing Systems. Association for Computing Machinery, New York, NY, USA, 1-6. 
[32] Ching Liu, Chi-Lan Yang, Joseph Jay Williams, and Hao-Chuan Wang. 2019. NoteStruct: Scaffolding Note-taking while Learning from Online Videos. In Extended Abstracts of the 2019 CHI Conference on Human Factors in Computing Systems. Association for Computing Machinery, New York, NY, USA, 1-6.

[33] Pam A Mueller and Daniel M Oppenheimer. 2014. The pen is mightier than the keyboard: Advantages of longhand over laptop note taking. Psychological science 25, 6 (2014), 1159-1168.

[34] Aditi S. Muralidharan, Zoltán Gyöngyi, and Ed Chi. 2012. Social annotations in web search. In CHI Conference on Human Factors in Computing Systems, CHI '12, Austin, TX, USA - May 05 - 10, 2012, Joseph A. Konstan, Ed H. Chi, and Kristina Höök (Eds.). Association for Computing Machinery, New York, NY, USA, 1085-1094.

[35] Minoru Nakayama, Kouichi Mutsuura, and Hiroh Yamamoto. 2017. Effectiveness of student's note-taking activities and characteristics of their learning performance in two types of online learning. International fournal of Distance Education Technologies (IFDET) 15, 3 (2017), 47-64.

[36] Les Nelson, Christoph Held, Peter Pirolli, Lichan Hong, Diane J. Schiano, and Ed H. Chi. 2009. With a little help from my friends: examining the impact of social annotations in sensemaking tasks. In Proceedings of the 27th International Conference on Human Factors in Computing Systems, CHI 2009, Boston, MA, USA, April 4-9, 2009, Dan R. Olsen Jr., Richard B. Arthur, Ken Hinckley, Meredith Ringel Morris, Scott E. Hudson, and Saul Greenberg (Eds.). Association for Computing Machinery, New York, NY, USA, 1795-1798.

[37] Marie K Norman. 2017. Twelve tips for reducing production time and increasing long-term usability of instructional video. Medical teacher 39, 8 (2017), 808-812.

[38] Jane Oakhill and Anne-Marie Davies. 1991. The effects of test expectancy on quality of note taking and recall of text at different times of day. British fournal of Psychology 82, 2 (1991), 179-189.

[39] Donald L Peters. 1972. Effects of note taking and rate of presentation on short-term objective test performance. Fournal of Educational Psychology 63, 3 (1972), 276.

[40] N Andrew Peterson, Paul W Speer, and David W McMillan. 2008. Validation of a brief sense of community scale: Confirmation of the principal theory of sense of community. Journal of community psychology 36, 1 (2008), 61-73.

[41] Stephen T Peverly, Joanna K Garner, and Pooja C Vekaria. 2014. Both handwriting speed and selective attention are important to lecture note-taking. Reading and Writing 27, 1 (2014), 1-30.

[42] Stephen T Peverly, Pooja C Vekaria, Lindsay A Reddington, James F Sumowski, Kamauru R Johnson, and Crystal M Ramsay. 2013. The relationship of handwriting speed, working memory, language comprehension and outlines to lecture note-taking and test-taking among college students. Applied Cognitive Psychology 27, 1 (2013), 115-126.

[43] Annie Piolat, Thierry Olive, and Ronald T Kellogg. 2005. Cognitive effort during note taking. Applied cognitive psychology 19, 3 (2005), 291-312.

[44] Eric D Ragan, Samuel R Jennings, John D Massey, and Peter E Doolittle. 2014. Unregulated use of laptops over time in large lecture classes. Computers \& Education 78 (2014), 78-86.

[45] Evan F Risko, Dawn Buchanan, Srdan Medimorec, and Alan Kingstone. 2013. Everyday attention: Mind wandering and computer use during lectures. Computers \& Education 68 (2013), 275-283.

[46] Daniel L Schacter and Karl K Szpunar. 2015. Enhancing attention and memory during video-recorded lectures. Scholarship of Teaching and Learning in Psychology 1, 1 (2015), 60.

[47] Tae Eun Shim and Song Yi Lee. 2020. College students' experience of emergency remote teaching due to COVID-19. Children and youth services review 119 (2020), 105578

[48] Hyungyu Shin, Eun-Young Ko, Joseph Jay Williams, and Juho Kim. 2018. Understanding the effect of in-video prompting on learners and instructors. In Proceedings of the 2018 CHI conference on human factors in computing systems. Association for Computing Machinery, New York, NY, USA, 1-12.

[49] Min-Young Song. 2012. Note-taking quality and performance on an L2 academic listening test. Language Testing 29, 1 (2012), 67-89.

[50] Peggy Van Meter, Linda Yokoi, and Michael Pressley. 1994. College students' theory of note-taking derived from their perceptions of note-taking. Journal of Educational Psychology 86, 3 (1994), 323.

[51] Hao-Chuan Wang, Cheng-Hsien Han, Mei-Hua Pan, and Chi-Lan Yang. 2016. How Social Annotation Affects Second Language Reading. In Proceedings of the 19th ACM Conference on Computer Supported Cooperative Work and Social Computing, CSCW 2015, San Francisco, CA, USA, February 27 - March 2, 2016, Companion Volume, Darren Gergle, Meredith Ringel Morris, Pernille Bjørn, and Joseph A. Konstan (Eds.). Association for Computing Machinery, New York, NY, USA, 429-432.

[52] Claire E Weinstein and Richard E Mayer. 1983. The teaching of learning strategies.. In Innovation abstracts, Vol. 5. ERIC, 4

[53] Joseph Jay Williams, Tania Lombrozo, Anne Hsu, Bernd Huber, and Juho Kim. 2016. Revising learner misconceptions without feedback: Prompting for reflection on anomalies. In Proceedings of the 2016 CHI Conference on Human Factors in Computing Systems. Association for Computing Machinery, New York, NY, USA, 470-474. 
[54] Robert L Williams and Alan C Eggert. 2002. Notetaking in college classes: Student patterns and instructional strategies. The fournal of General Education 51, 3 (2002), 173-199.

[55] Barry J Zimmerman and Dale H Schunk. 2011. Handbook of self-regulation of learning and performance. Taylor \& Francis Group.

Received July 2021; revised September 2021; accepted October 2021 